RESEARCH SERIES

$\begin{array}{lc}\text { NISTIR 89-4189 } & \text { NIST } \\ & \text { PUBLICATIONS }\end{array}$

October 5, 1989

\section{AMRF PART MODEL EXTENSIONS}

By:

Allison Barnard

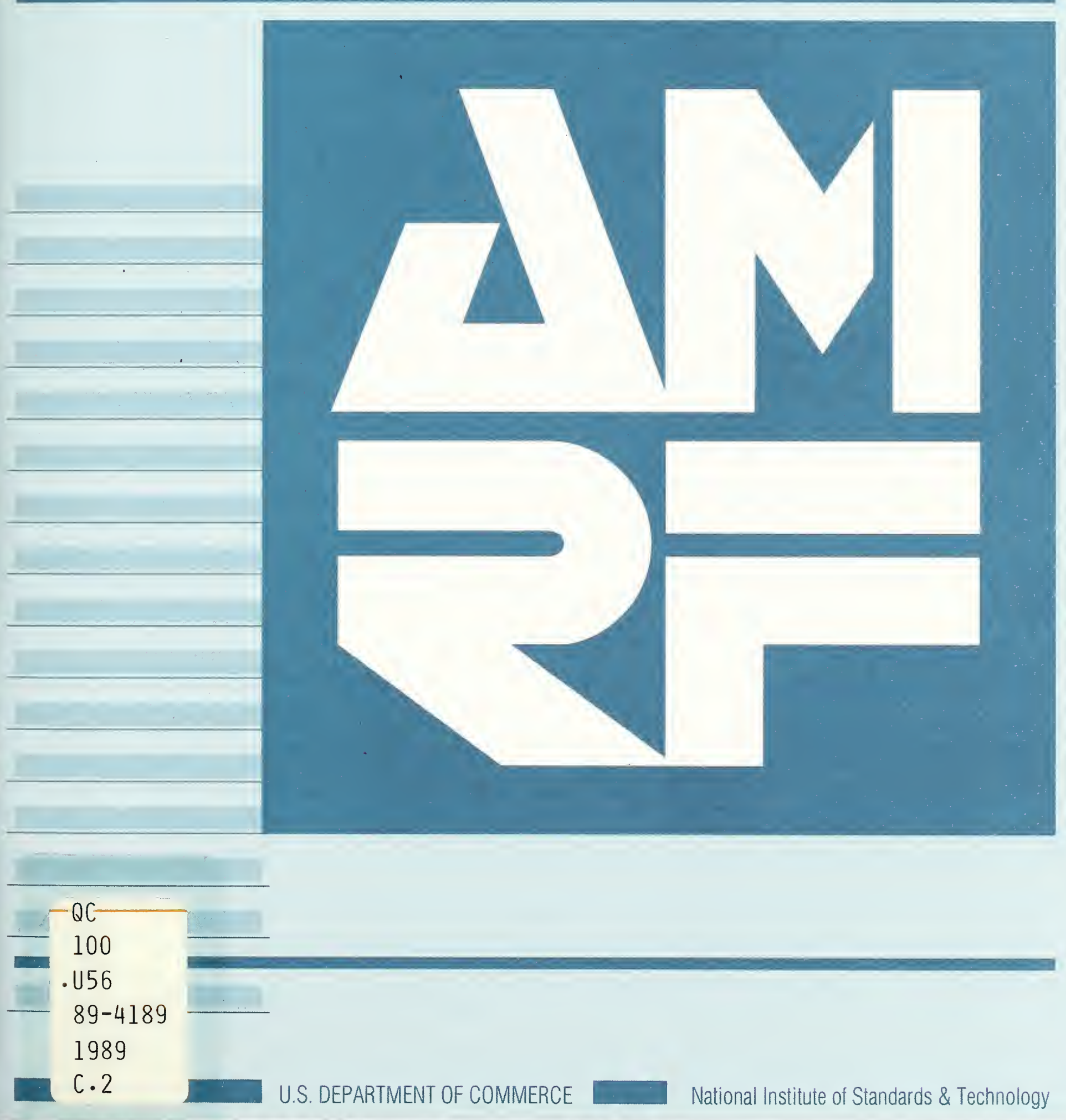


NATIONAL INSTITUTE OF STANDARDS \&

TECHNOLOGY

Research Information Center

Gaithersburg, MD 20899

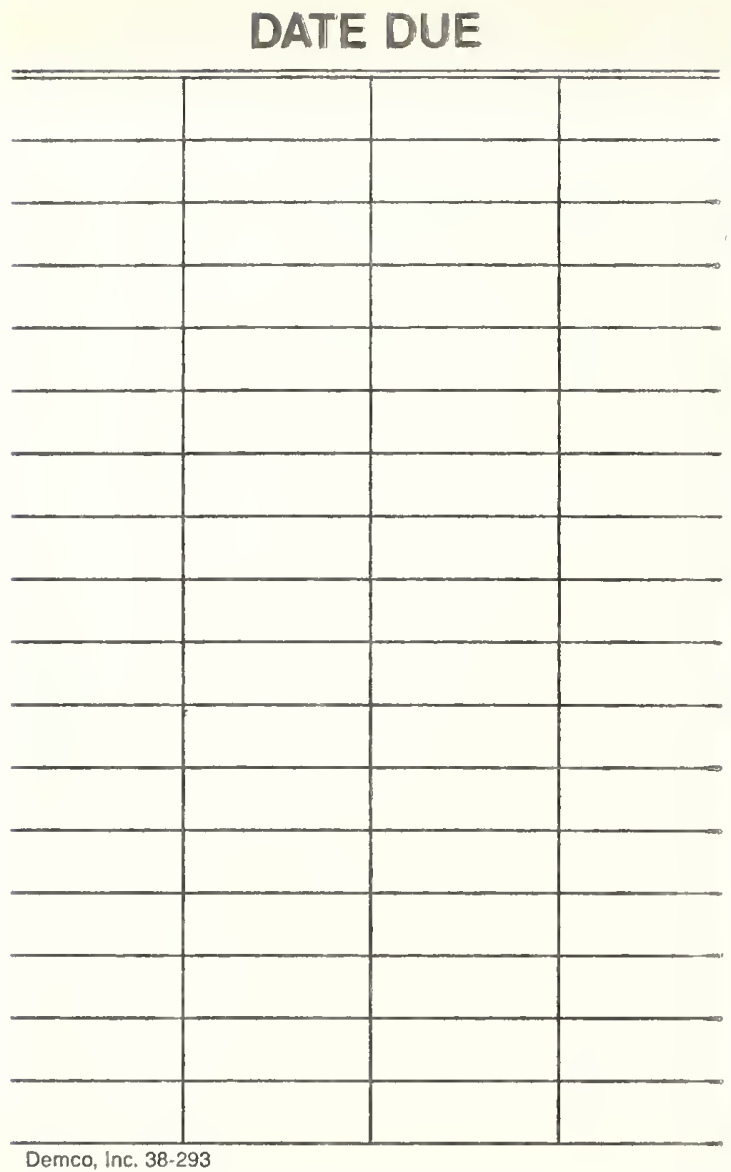




\title{
AMRF Part Model Extensions
}

\author{
Allison Barnard \\ October 1989
}

This publication was prepared by United States Government Employees as part of their official duties and is, therefore, a work of the U. S. Government and not subject to copyright.

Certain commercial equipment, instruments, or materials are identified in this paper in order to adequately specify the experimental procedure. Such identification does not imply recommendation or endorsement by the National Institute of Standards and Technology, nor does it imply that the materials or equipment identified are necessarily the best available for the purpose. 



\section{TABLE OF CONTENTS}

1. INTRODUCTION 1

1.1 Motivation 1

1.2 Audience 1

1.3 Overview 1

2. PART MODEL EXTENSIONS 2

2.1 Structure of the Part Model Format 2

2.2 Explanation of Formal Language Syntax 2

2.3 Use of the Part Model Extensions 3

APPENDICES 4

APPENDIX A. GRAMMAR 4

APPENDIX B. DICTIONARY STRUCTURES 6

APPENDIX C. TYPE DEFINITIONS

APPENDIX D. SAMPLE PART MODEL FILES 9

$\begin{array}{ll}\text { REFERENCES } & 26\end{array}$ 


\section{LIST OF FIGURES}

Figure 1. Topology and Geometry Hierarchy in a Boundary Model 2

Figure 2. Sketch of Solid Model Containing Ellipses 9

Figure 3. Sketch of Solid Model Containing Hyperbolas 14

Figure 4. Torpedo Motor Containing B-Splines 20 


\section{AMRF Part Model Extensions}

\section{INTRODUCTION}

\subsection{Motivation}

The AMRF part model format was created as a data specification language for the communication of product data between manufacturing application processes and the global database within the Automated Manufacturing Research Facility [Barkmeyer86]. The part model report format was intended to be a temporary solution to a subset of the complex problems in automating manufacturing that were not being addressed by the current standard Initial Graphics Exchange Specification (IGES). The experience gained through the implementation of the format was to be used as input for developing national standards [Hopp87a] such as ISO's Product Data Exchange Specification (PDES) [Smith88].

This document specifies the addition of coordinate system independent representations of ellipses, hyperbolas, parabolas and non-rational, uniform b-splines to the part model report. These changes were the recommendation of a Manufacturing Data Preparation project working group [Fowler88]. While the addition of these entities does not resolve all the shortcomings of the format, it was necessary to allow the use of more complex test parts within the AMRF. Further geometric extensions will result from the migration to PDES.

\subsection{Audience}

This document is a reference guide to the grammar of the extensions to the AMRF part model. This document is intended to be used by programmers implementing systems that will make use of AMRF part model data. It is expected that the user is familiar with the contents of the document "AMRF Database Report Format: Part Model" [Hopp87b] as this document contains only the additions to the part model format. The two documents must be used in conjunction with each other for completeness.

\subsection{Overview}

Section 2 of this document describes the structure and syntax of the part model report grammar and mentions tools that use the format. Appendix A provides a formal 
definition of the new grammar. Appendix B contains the revised dictionary definition. Appendix $\mathrm{C}$ provides a formal definition of the new data types. Appendix $\mathrm{D}$ contains sample part model files along with sketches of the parts being modeled.

\section{PART MODEL EXTENSIONS}

\subsection{Structure of the Part Model Format}

The AMRF part model format has five main sections; header, topology, geometry, features and functionality. At minimum, the report must contain the geometry and topology of a valid boundary representation solid model. Figure 1 illustrates the structure of the geometry and topology hierarchy. Ellipse, hyperbola, parabola and b-spline all fall under the curves subsection of the geometry section of the part model format.

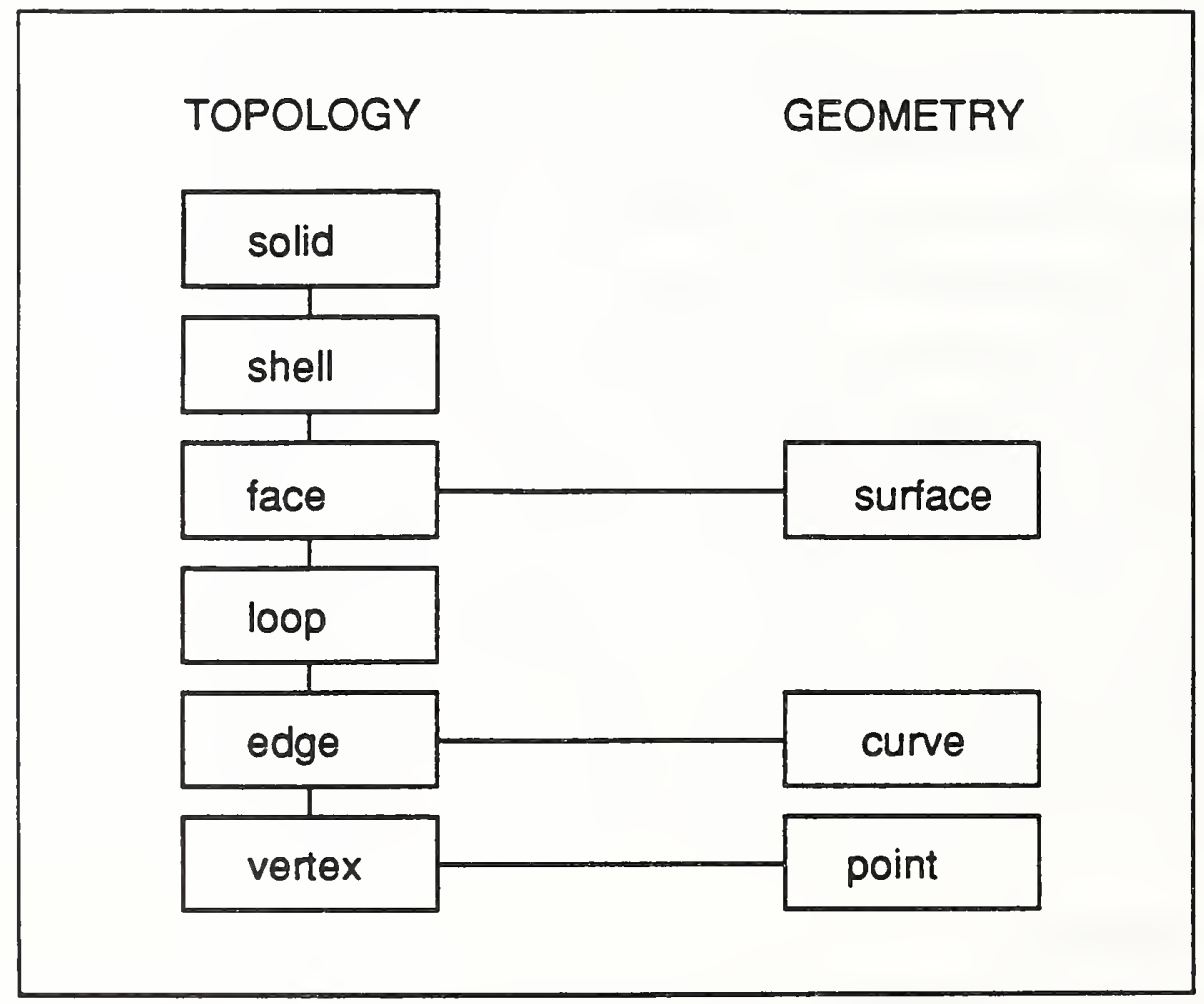

Figure 1. Topology and Geometry Hierarchy in a Boundary Model [Hopp87b]

\subsection{Explanation of Formal Language Syntax}

The additions, like the rest of the part model grammar, are expressed in Backus Naur Form (BNF); a context free syntax. This grammar has been annotated with semantic rules that define how the reports should be interpreted when parsed. The semantic rules express the standard AMRF interpretation for these reports. As such, these rules should be viewed as a guide, rather than a requirement as to how the parser should be implemented. 
The syntax rules are of the form:

$$
\text { left-hand-side ::= right-hand-side }
$$

where the left-hand-side is a single non-terminal symbol in the grammar and the right-handside is a sequence of one or more grammar symbols. Grammar symbols in the right-hand side that are to appear verbatim in the report appear in boldface type. All other symbols are enclosed in angle brackets <like this>. Terminal symbols other than literals are underlined. Numbers are the only terminal symbols that appear in the extensions, they have a val attribute that represents the value of the number.

Semantic rules are associated with each reduction in the grammar. They define how attributes from the left-hand-side of a reduction should be computed from attributes of the right-hand-side syntactic elements. The semantic rules are divided into two parts: the first labeled Semantics defines the actions that should be taken when a particular reduction is applied during the parsing. The Constraints section defines consistency criteria that can be used at the time a reduction is applied to check for errors in the report.

The semantic rules make use of global dictionary data structures. Dictionaries may be any sort of convenient data structure, from a linked list to a hash table to a relational database. They function as symbol tables for objects named in the report. A dictionary should be considered an array of identical elements indexed by symbolic names. The extensions defined in this report specify new entity structures for curveDictionary. The semantic rules refer to entities in pointDictionary and unitVectorDictionary. Entities in all dictionaries are named entities; entities in different dictionaries in the same part may have the same name, while still being distinct entities. For further details see [Hopp87b].

\subsection{Use of the Part Model Extensions}

Several tools exist which support the AMRF part model format. Manufacturing applications within the AMRF receive information from the part model through a parser which treats the report as a source of data and creates a set of initialized data structures in memory. An updated version of the part model parser exists and can be invoked by following the directions found in "Using the AMRF Part Model Format" [Ressler87]. Prototype part model report generation software has been implemented on several publicly available CAD systems at NIST. Solid model representations of parts created on these systems may be extracted from the proprietary database and converted to the AMRF part model format. The part model report may then be translated into a subset of PDES/STEP by another translator. For details on PDES conversion, see "Converting the AMRF Part Model Report to a PDES/STEP Subset" [Lee88]. 


\section{APPENDIX A: GRAMMAR}

1. <curve-description> ::= ELLIPSE; <point-id $>$; $<$ unit-vector-id $\rangle_{1}$; $<$ unit-vector-id $>_{2}$; $<$ number $_{1}$; $<$ number $_{2}$.

Semantics:

curve (<curve-description>)<--

ELLIPTICAL_CURVE(

center: pointDictionary [name $(<$ point-id $>)$ ],

major_axis: unitVectorDictionary [name(<unit-vector-id $\left.>_{1}\right)$ ],

normal: unitVectorDictionary [name (<unit-vector-id $\left.>_{2}\right)$ ],

major_radius: $\left.\operatorname{val}(<\underline{\text { number }}\rangle_{1}\right)$,

minor_radius: $\left.\operatorname{val}\left(<\underline{\text { number }}_{2}\right)\right)$

Constraints:

If $\left.\mathrm{d}_{1}=\operatorname{dir}(\text { unitVectorDictionary [name (<unit-vector-id }\rangle_{1}\right)$ ])

$\mathrm{d}_{2}=\operatorname{dir}\left(\right.$ unitVectorDictionary [name $\left(<\right.$ unit-vector-id $\left.\left.>_{2}\right)\right]$ )

Then $d_{1} \cdot d_{2}=0$

$<$ number $>_{1},<\underline{\text { number }}>_{2}>0$

2. <curve-description> ::= HYPERBOLA; < point-id>; <unit-vector-id $>_{1}$; <unit-vectorid $>_{2} ;<$ number $>_{1} ;<$ number $>_{2}$.

Semantics:

curve (<curve-description>)<--

HYPERBOLIC_CURVE(

center: pointDictionary [name $(<$ point-id $>)]$,

major_axis: unitVectorDictionary [name $\left(<\right.$ unit-vector-id $\left.>_{1}\right)$ ],

normal: unitVectorDictionary [name $\left(<\right.$ unit-vector-id $\left.>_{2}\right)$ ],

major_radius: $\operatorname{val}\left(<\underline{\text { number }}>_{1}\right)$,

minor_radius: $\left.\operatorname{val}\left(<\underline{\text { number }}>_{2}\right)\right)$

Constraints:

If $\mathrm{d}_{1}=\operatorname{dir}\left(\right.$ unitVectorDictionary [name $\left(<\right.$ unit-vector-id $\left.\left.>_{1}\right)\right]$ )

$\mathrm{d}_{2}=\operatorname{dir}\left(\right.$ unitVectorDictionary [name $\left(<\right.$ unit-vector-id $\left.\left.{ }_{2}\right)\right]$ )

Then $\mathrm{d}_{1} \cdot \mathrm{d}_{2}=0$

$<$ number $>_{1},<$ number $>_{2}>0$

The branch of the hyperbola represented is that pointed to by the major_axis.

3. <curve-description> ::= PARABOLA; < id $>2 ;$ number $>$.

Semantics: 


\section{Constraints:}

curve (<curve-description>) <--

\section{PARABOLIC_CURVE(}

center: pointDictionary [name $(<$ point-id $>)$ ],

focal_dir: unitVectorDictionary [name $\left(<\right.$ unit-vector-id $\left.>_{1}\right)$ ],

normal: unitVectorDictionary [name $\left(<\right.$ unit-vector-id $\left.>_{2}\right)$ ],

focal_dist: $v a l(<\underline{\text { number }}>))$

If $\mathrm{d}_{1}=\operatorname{dir}\left(\right.$ unitVectorDictionary [name (<unit-vector-id $\left.\left.>_{1}\right)\right]$ )

$\mathrm{d}_{2}=\operatorname{dir}\left(\right.$ unitVectorDictionary [name $\left(<\right.$ unit-vector-id $\left.\left.>_{2}\right)\right]$ )

Then $d_{1} \cdot d_{2}=0$

$<$ number $><>0$

4. $<$ curve-description $>::=\mathbf{B}-$ SPLINE; $<\underline{\text { number }}\rangle_{1}$; $\left.<\underline{\text { number }}\right\rangle_{2}$; $<$ point-id-list $>$.

Semantics:

curve (<curve-description $>$ ) <--

B-SPLINE_CURVE(

degree: $\operatorname{val}\left(<\underline{\text { number }}>_{1}\right)$,

number_control_points: val $\left(<\underline{\text { number }}>_{2}\right)$,

Constraints:

control_points: points(<point-id-list $>)$ )

$$
\begin{aligned}
& \operatorname{val}\left(<\text { number }_{1}\right) \text { is an integer }>=0 \\
& \operatorname{val}\left(<\underline{\text { number }}>_{2}\right) \text { is an integer }>=\left(\operatorname{val}\left(\left\langle\underline{\text { number }}>_{1}\right)+1\right)\right. \\
& <\text { point-id-list }>\text { is an array }\left[1 . \text { val }\left(<\text { number }_{2}\right)\right] \text { of points }
\end{aligned}
$$

5. $<$ point-id-lis $>::=<$ point-id $>$

Semantics:

points(<point-id-list $>)<-$

$$
\text { LIST( }
$$

head: pointDictionary[name $(<$ point-id $>)$ ],

Constraints: NONE tail: nil)

6. $\langle\text { point-id-list }\rangle_{1}::=\langle$ point-id $\rangle,\langle\text { point-id-lis }\rangle_{2}$

Semantics:

$$
\text { points }\left(<\text { point-id-list }>_{1}\right)<--
$$

LIST(

head: pointDictionary[name $(<$ point-id $>)$ ], tail: points $\left.(<\text { point-id-list }\rangle_{2}\right)$ )

Constraints: NONE 


\section{APPENDIX B: DICTIONARY STRUCTURES}

curveDictionary

index: curve id string

contents:

name: curve id string

curve: a curve description

A curve description can now be one of ELLIPTICAL_CURVE, HYPERBOLIC_CURVE, PARABOLIC_CURVE, B-SPLINE_CURVE data structures in addition to the LINEAR_CURVE and CIRCULAR_CURVE data structures previously allowed.

The following dictionary structures are unchanged but are included for completeness:

pointDictionary

index: point id string

contents:

name: point id string

vec: a vector

A vector is a VECTOR data structure.

unit VectorDictionary

index: unit vector id string

contents:

name: unit vector id string

dir: a direction

A direction is a UNIT_VECTOR data structure. 


\section{APPENDIX C: TYPE DEFINITIONS}

\section{ELLIPTICAL_CURVE}

A structure with five fields:

center: a point

major_axis: a direction

normal: a direction

major_radius: a number

minor_radius: a number

A point data structure is an entry in pointDictionary. A direction data structure is an entry in the unitVectorDictionary.

\section{HYPERBOLIC CURVE}

A structure with five fields:

center: a point

major_axis: a direction

normal: a direction

major_radius: a number

minor_radius: a number

A point data structure is an entry in pointDictionary. A direction data structure is an entry in the unitVectorDictionary.

\section{PARABOLIC_CURVE}

A structure with four fields:

center: a point

focal_dir: a direction

normal: a direction

focal_dist: a number

A point data structure is an entry in pointDictionary. A direction data structure is an entry in the unitVectorDictionary.

\section{B-SPLINE CURVE}

A structure with three fields:

degree: a number

number_control_points: a number

control_points: a list of points

A point data structure is an entry in pointDictionary. 
The type LIST was used in "AMRF Database Report Format: Part Model" but was omitted from the type definitions. It is included here for the sake of completeness.

\section{LIST}

A LIST is either the constant nil or a structure with two fields:

head: an element from a dictionary

tail: a LIST

The data structure of the head is determined by the dictionary in which the element occurs.

The types VECTOR and UNIT_VECTOR are unchanged but are included here for the completeness.

\section{VECTOR}

A structure with three fields:
$\mathrm{x}$ : a number
y: a number
z: a number

\section{UNIT_VECTOR}

A structure with three fields:
$\mathrm{x}$ : a number
$y:$ a number
z: a number 


\section{APPENDIX D: SAMPLE PART MODEL FILES}

The following examples are included to illustrate the use of the extensions. To test the implementation of these extensions, solid models containing the curves were created on the Computervision CAD system. A prototype extraction tool developed in-house was then used to convert between Computervision's proprietary database and the AMRF part model report format. In the text, the curve representations have been emboldened.

\section{Ellipse}

The part ELLIPSE_TEST was created by subtracting a block from a cylinder such that the block intersected the curved surface at an angle. There are four distinct ellipses represented in this model. The part model file follows Figure 2.
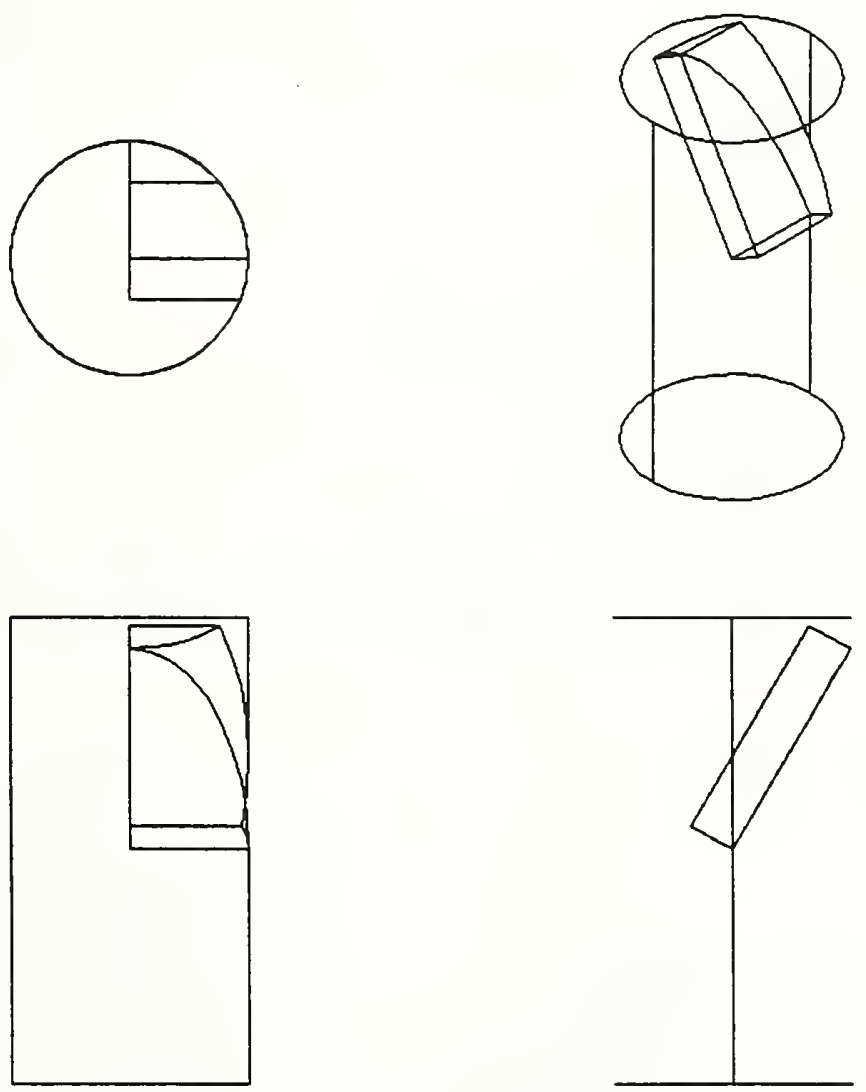

Figure 2. Sketch of Solid Model Containing Ellipses 
/PART_MODEL

/HEADER

PART_NAME = 'ELLIPSE_TEST'.

/END_HEADER

ITOPOLOGY

ISHELLS

SHL1;FAC280,FAC281,FAC282,FAC284,FAC283,FAC285,FAC286, FAC295,FAC297.

IEND_SHELLS

IFACES

FAC280;LOP28001; SRF288 + .

FAC281;LOP28101; SRF289 + .

FAC282;LOP28201; SRF290 + .

FAC284;LOP28401; SRF291 + .

FAC283;LOP28301; SRF292 + .

FAC285;LOP28501; SRF293 + .

FAC286;LOP28601; SRF294 + .

FAC295;LOP29501; SRF296 + .

FAC297;LOP29701; SRF298 + .

/END_FACES

LOOPS

LOP28001;EDG301-,EDG264+,EDG270-,EDG299+.

LOP28101;EDG266+,EDG299-,EDG268+,EDG276+.

LOP28201;EDG268-,EDG270+,EDG279+.

LOP28401;EDG264-,EDG262+,EDG278-.

LOP28301;EDG277-,EDG262-,EDG301+,,EDG266-,EDG275-.

LOP28501;EDG276-,EDG272-,EDG307+,EDG305+,EDG303-,EDG274-,EDG275+.

LOP28601;EDG274+,EDG311-,EDG305-,EDG309+,EDG272+,EDG279-,EDG278+,

EDG277+.

LOP29501;EDG303+,EDG311+.

LOP29701;EDG309-,EDG307-.

/END_LOOPS

/EDGES

EDG299;VTX316,VTX315; CRV300 +.

EDG270;VTX316,VTX260; CRV269 +.

EDG264;VTX317,VTX260; CRV263 +.

EDG301;VTX317,VTX315; CRV302 +.

EDG276;VTX258,VTX257; CRV252 +.

EDG268;VTX316,VTX258; CRV267 +.

EDG266;VTX257,VTX315; CRV265 +.

EDG279;VTX260,VTX258; CRV255 +. 


$$
\begin{aligned}
& \text { EDG278;VTX260,VTX256; CRV254 +. } \\
& \text { EDG262;VTX317,VTX256; CRV261 +. } \\
& \text { EDG275;VTX259,VTX257; CRV251 +. } \\
& \text { EDG277;VTX256,VTX259; CRV253 +. } \\
& \text { EDG274;VTX259,VTX319; CRV273 +. } \\
& \text { EDG303;VTX319,VTX318; CRV304 +. } \\
& \text { EDG305;VTX314,VTX318; CRV306 +. } \\
& \text { EDG307;VTX313,VTX314; CRV308 +. } \\
& \text { EDG272;VTX313,VTX258; CRV271 +. } \\
& \text { EDG309;VTX314,VTX313; CRV310 +. } \\
& \text { EDG311;VTX318,VTX319; CRV312 +. }
\end{aligned}
$$

/END_EDGES

NERTICES

VTX316;PNT3160.

VTX315;PNT3150.

VTX260;PNT2600.

VTX317;PNT3170.

VTX258;PNT2580.

VTX257;PNT2570.

VTX256;PNT2560.

VTX259;PNT2590.

VTX319;PNT3190.

VTX318;PNT3180.

VTX314;PNT3140.

VTX313;PNT3130.

/END_VERTICES

/END_TOPOLOGY

/GEOMETRY

ISURFACES

SRF288; PLANE; UNV2650; 0.00000000.

SRF289; PLANE; UNV2510; 0.00000000 .

SRF290; PLANE; UNV2540; 0.00000000 .

SRF291; PLANE; UNV2521; 10.00000000 .

SRF292; PLANE; UNV3000; 2.00000000 .

SRF293; CYLINDER; PNT3080; UNV2730; 5.00000000.

SRF294; CYLINDER; PNT3080; UNV2730; 5.00000000.

SRF296; PLANE; UNV2730; 10.00000000 .

SRF298; PLANE; UNV2730; -10.00000000 .

/END_SURFACES

ICURVES 
CRV300; LINE; PNT3160; UNV3000.

CRV269; LINE; PNT3160; UNV2690.

CRV263; LINE; PNT3170; UNV2630.

CRV302; LINE; PNT3170; UNV3020.

CRV252; ELLIPSE; PNT3160; UNV3000; UNV2521; 5.77350283; 5.00000000.

CRV267; LINE; PNT3160; UNV2670.

CRV265; LINE; PNT2570; UNV2650.

CRV255; ELLLIPSE; PNT2550; UNV2521; UNV3000; $10.00000000 ; 5.00000000$. CRV254; ELLIIPSE; PNT2540; UNV2540; UNV2521; 5.77350283; 5.00000000 . CRV261; LINE; PNT3170; UNV2670.

CRV251; ELLIPSE; PNT2510; UNV2510; UNV3000; $10.00000000 ; 5.00000000$. CRV253; ELLIPSE; PNT2510; UNV2510; UNV3000; $10.00000000 ; 5.00000000$. CRV273; LINE; PNT2590; UNV2730.

CRV304; CIRCLE; PNT3040; UNV2730; PNT3190.

CRV306; LINE; PNT3140; UNV2730.

CRV308; CIRCLE; PNT3080; UNV2730; PNT3130.

CRV271; LINE; PNT3130; UNV2730.

CRV310; CIRCLE; PNT3080; UNV2730; PNT3101.

CRV312; CIRCLE; PNT3040; UNV2730; PNT3121.

IEND_CURVES

IPOINTS

PNT3160; $0.00000000,0.00000000,0.00000000$.

PNT3150; $0.00000000,-1.00000000,1.73205078$.

PNT2600; $0.00000000,-8.66025448,-5.00000000$.

PNT3170; $0.00000000,-9.66025448,-3.26794910$.

PNT2580; $5.00000000,0.00000046,0.00000000$.

PNT2570; 4.69041586, -1.00000000, 1.73205078 .

PNT2560; $3.78424478,-9.66025448,-3.26794910$.

PNT2590; 5.00000000, -3.99999952, 0.00000000 .

PNT3190; $5.00000000,-10.00000000,0.00000000$.

PNT3180; $-5.00000000,-10.00000000,0.00000000$

PNT3140; $-5.00000000,10.00000000,0.00000000$.

PNT3130; $5.00000000,10.00000000,0.00000000$.

PNT2550; $0.00000000,-0.00000030,0.00000000$.

PNT2540; $0.00000000,-11.54700565,0.00000000$.

PNT2510; $0.00000000,-4.00000048,0.00000000$.

PNT3040; $0.00000000,-10.00000000,0.00000000$.

PNT3080; $0.00000000,10.00000000,0.00000000$.

PNT3101; $-5.00000000,10.00000000,-0.00000044$.

PNT3121; $-5.00000000,-10.00000000,-0.00000044$. 


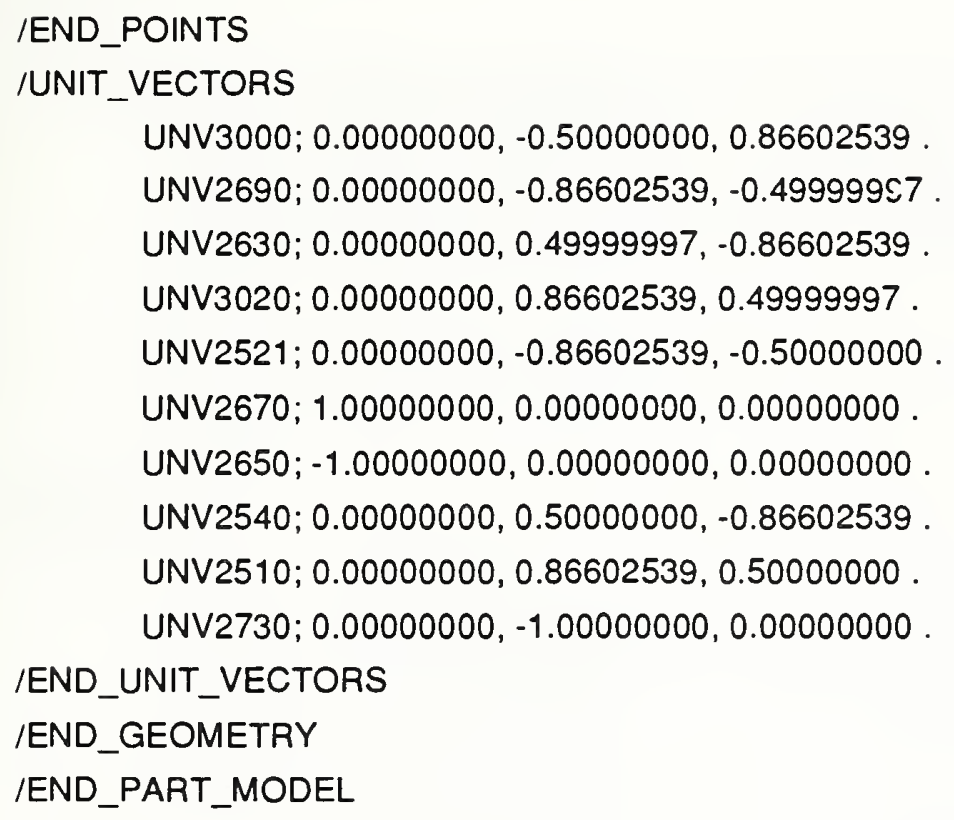




\section{Hyperbola}

The part HYPERBOLA_TEST was modeled by subtracting a block from a cone where the sides of the block were parallel to and as long as the axis of the cone. There are four distinct hyperbolas in the model. The complete part model file follows the illustration in Figure 3.

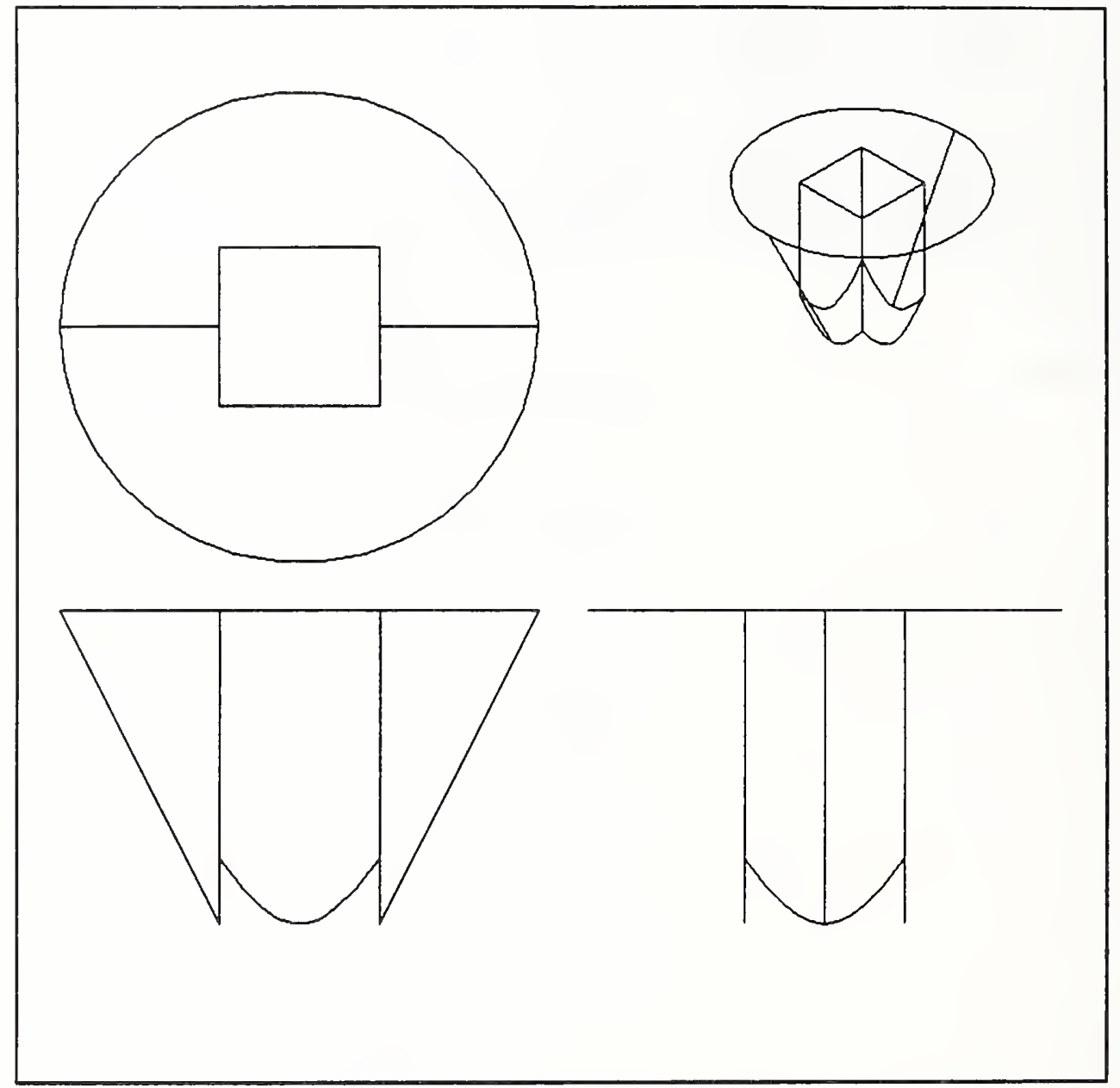

Figure 3. Sketch of Solid Model Containing Hyperbolas

/PART_MODEL

IHEADER

PART_NAME $=$ 'HYPERBOLA_TEST'.

IEND_HEADER

ITOPOLOGY

ISHELLS

SHL1;FAC2075,FAC2081,FAC2076,FAC2077,FAC2078,FAC2079,FAC2080.

/END_SHELLS 
/FACES

FAC2075;LOP207501; SRF2083 + .

FAC2081;LOP208101; SRF2084 + .

FAC2076;LOP207601; SRF2085 + .

FAC2077;LOP207701,LOP207702; SRF2086 + .

FAC2078;LOP207801; SRF2087 + .

FAC2079;LOP207901; SRF2088 + .

FAC2080;LOP208001; SRF2089 + .

IEND_FACES

ILOOPS

LOP207501;EDG2074-,EDG2073-,EDG2054-,EDG2090+,EDG2056+,EDG2072- .

LOP208101;EDG2062-,EDG2072+,EDG2069+,EDG2064-,EDG2065+.

LOP207601;EDG2056-,EDG2092+,EDG2054+,EDG2071-,EDG2070-,EDG2069-.

LOP207701;EDG2090-,EDG2092-.

LOP207702;EDG2066+,EDG2067-,EDG2068-,EDG2065-.

LOP207801;EDG2060-,EDG2071+,EDG2073+,EDG2058-,EDG2067+.

LOP207901;EDG2058+,EDG2074+,EDG2062+,EDG2068+.

LOP208001;EDG2064+,EDG2070+,EDG2060+,EDG2066-.

/END_LOOPS

/EDGES

EDG2072;VTX2049,VTX2044; CRV2040 +. EDG2056;VTX2095,VTX2044; CRV2055 +. EDG2090;VTX2094,VTX2095; CRV2091 +. EDG2054;VTX2094,VTX2043; CRV2053 +. EDG2073;VTX2043,VTX2046; CRV2041 +. EDG2074;VTX2046,VTX2049; CRV2042 +. EDG2065;VTX2051,VTX2050; CRV2033 +. EDG2064;VTX2051,VTX2052; CRV2063 +. EDG2069;VTX2044,VTX2052; CRV2037 +. EDG2062;VTX2049,VTX2050; CRV2061 +. EDG2070;VTX2052,VTX2047; CRV2038 +. EDG2071;VTX2047,VTX2043; CRV2039 +. EDG2092;VTX2095,VTX2094; CRV2093 +. EDG2068;VTX2050,VTX2045; CRV2036 +. EDG2067;VTX2045,VTX2048; CRV2035 +. EDG2066;VTX2051,VTX2048; CRV2034 +. EDG2058;VTX2045,VTX2046; CRV2057 +. EDG2060;VTX2047,VTX2048; CRV2059 +.

/END_EDGES

IVERTICES 
VTX2049;PNT20490.

VTX2044;PNT20440.

VTX2095;PNT20950.

VTX2094;PNT20940.

VTX2043;PNT20430.

VTX2046;PNT20460.

VTX2051;PNT20510.

VTX2050;PNT20500.

VTX2052;PNT20520.

VTX2047;PNT20470.

VTX2045;PNT20450.

VTX2048;PNT20480.

/END_VERTICES

/END_TOPOLOGY

IGEOMETRY

ISURFACES

SRF2083; CONE; PNT20831; UNV20910; 0.89442718.

SRF2084; PLANE; UNV20401; 1.00000000 .

SRF2085; CONE; PNT20831; UNV20910; 0.89442718.

SRF2086; PLANE; UNV20910; -6.00000000 .

SRF2087; PLANE; UNV20411; 1.00000000 .

SRF2088; PLANE; UNV20421; 1.00000000 .

SRF2089; PLANE; UNV20381; 1.00000000 .

/END_SURFACES

ICURVES

CRV2040; HYPERBOLA; PNT20400; UNV20400; UNV20401;

$2.00000000 ; 1.00000000$.

CRV2055; LINE; PNT20950; UNV20550.

CRV2091; CIRCLE; PNT20910; UNV20910; PNT20940.

CRV2053; LINE; PNT20940; UNV20530.

CRV2041; HYPERBOLA; PNT20410; UNV20400; UNV20411;

2.00000000; 1.00000000 .

CRV2042; HYPERBOLA; PNT20420; UNV20400; UNV20421;

2.00000000; 1.00000000 .

CRV2033; LINE; PNT20330; UNV20421.

CRV2063; LINE; PNT20510; UNV20910.

CRV2037; HYPERBOLA; PNT20400; UNV20400; UNV20401;

2.00000000; 1.00000000 .

CRV2061; LINE; PNT20490; UNV20400.

CRV2038; HYPERBOLA; PNT20380; UNV20400; UNV20381; 


\section{$2.00000000 ; 1.00000000$.}

CRV2039; HYPERBOLA; PNT20410; UNV20400; UNV20411;

\subsection{0; 1.00000000 .}

CRV2093; CIRCLE; PNT20910; UNV20910; PNT20950.

CRV2036; LINE; PNT20360; UNV20411.

CRV2035; LINE; PNT20350; UNV20381.

CRV2034; LINE; PNT20340; UNV20411.

CRV2057; LINE; PNT20450; UNV20910.

CRV2059; LINE; PNT20470; UNV20400.

\section{IEND_CURVES}

IPOINTS

PNT20490; -1.00000000, -2.82842708, -1.00000000.

PNT20440; $-1.00000000,-2.00000000,0.00000000$.

PNT20950; $-3.00000000,-6.00000000,0.00000000$.

PNT20940; 3.00000000, $-6.00000000,0.00000000$.

PNT20430; $1.00000000,-2.00000000,0.00000000$.

PNT20460; $1.00000000,-2.82842708,-1.00000000$.

PNT20510; $-1.00000000,-6.00000000,1.00000000$.

PNT20500; $-1.00000000,-6.00000000,-1.00000000$.

PNT20520; -1.00000000, -2.82842708, 1.00000000 .

PNT20470; $1.00000000,-2.82842708,1.00000000$.

PNT20450; $1.00000000,-6.00000000,-1.00000000$.

PNT20480; $1.00000000,-6.00000000,1.00000000$.

PNT20400; $-1.00000000,0.00000000,0.00000000$.

PNT20910; $0.00000000,-6.00000000,0.00000000$.

PNT20410; $1.00000000,0.00000000,0.00000000$.

PNT20420; $0.00000000,0.00000000,-1.00000000$.

PNT20330; $-1.00000000,-6.00000000,1.00000012$.

PNT20380; $0.00000000,0.00000000,1.00000000$.

PNT20360; -1.00000012, $-6.00000000,-1.00000000$.

PNT20350; $1.00000000,-6.00000000,-1.00000012$.

PNT20340; $-1.00000012,-6.00000000,1.00000000$.

PNT20831; $0.00000000,0.00000000,0.00000000$.

/END_POINTS

IUNIT_VECTORS

UNV20400; $0.00000000,-1.00000000,0.00000000$.

UNV20401; $-1.00000000,0.00000000,0.00000000$

UNV20550; $0.44721359,0.89442718,0.00000000$.

UNV20910;0.00000000, $1.00000000,0.00000000$.

UNV20530; $-0.44721359,0.89442718,0.00000000$. 
UNV20411; $1.00000000,0.00000000,0.00000000$ UNV20421; $0.00000000,0.00000000,-1.00000000$. UNV20381; $0.00000000,0.00000000,1.00000000$. /END_UNIT_VECTORS

IEND_GEOMETRY

/END_PART_MODEL 


\section{Parabola}

A parabolic intersection curve occurs when a plane is oriented such that the angle of the plane and the angle of the cone surface to the cone axis is the same. However, no Computervision solid models created in this manner contain parabolas. An example of the representation of a parabola is included in the place of a part model file:

CRV13300; PARABOLA; PNT132920; UNV117340; UNV121220; PNT132897. 


\section{B-Spline}

The part TORPEDO_MOTOR was also modeled on the Computervision system and is an example of an AMRF test part. The part model file for the torpedo motor is too long to be included in its entirety. This part is an example of the complexity of the parts that can now be handled with the AMRF part model format.

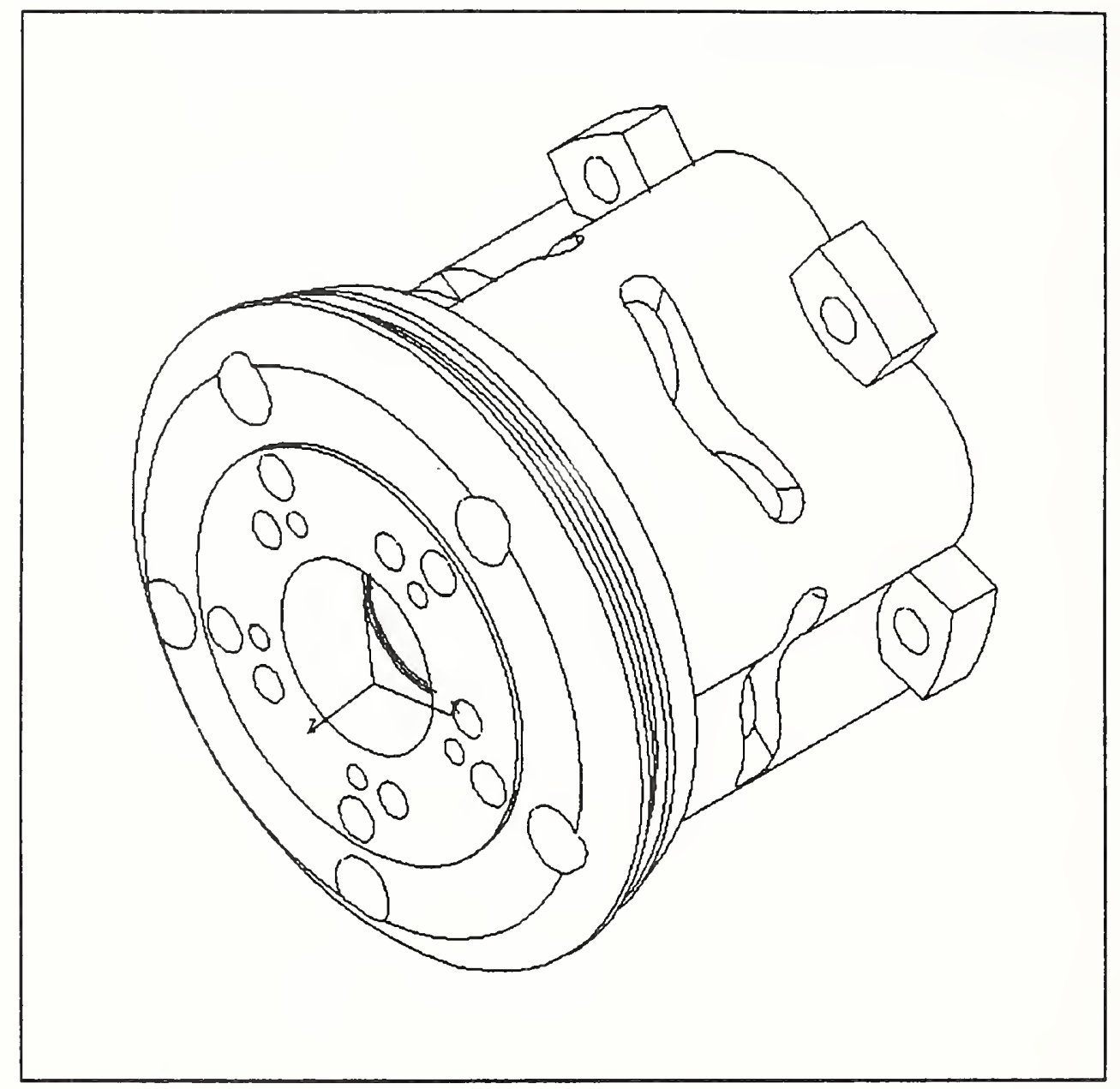

Figure 4. Torpedo Motor Containing B-Splines

PART_MODEL

IHEADER

PART_NAME = 'TORPEDO_MOTOR'.

IEND_HEADER

ITOPOLOGY

ISHELLS

SHL1;FAC11846,FAC11859,FAC11864,FAC11861,FAC11858,FAC11851,

FAC 11872,FAC11874,FAC11876,FAC11878,FAC11880,FAC11882,FAC11884,

FAC11886,FAC11888,FAC11890,FAC11892,FAC11894,FAC11850,FAC11847, 
FAC11862,FAC11855,FAC11854,FAC11863,FAC11852,FAC11848,FAC11849, FAC11853,FAC11860,FAC11857,FAC11856,FAC11909,FAC11911,FAC11913, FAC11915,FAC11917,FAC11919,FAC 11921,FAC11923,FAC11925,FAC11927, FAC12293,FAC12295,FAC12297,FAC12299, FAC12301, FAC12303,FAC12305, FAC 12307,FAC12309,FAC12311,FAC 12313,FAC12315,FAC12317,FAC12319, FAC12321,FAC12323,FAC12325,FAC 12327,FAC12329,FAC12331,FAC 12333, FAC12335,FAC12337,FAC12339,FAC12341,FAC12343,FAC12345,FAC12347, FAC12349,FAC12351,FAC12353,FAC12355,FAC12357,FAC12359,FAC12361, FAC12363,FAC12365,FAC12367,FAC12369,FAC12371,FAC12373,FAC12375.

/END_SHELLS

IFACES

FAC11846;LOP1184601; SRF11866 +.

FAC11859;LOP1185901; SRF11867 +.

FAC11864;LOP1186401; SRF11868 +.

FAC 11861;LOP1186101; SRF11869 +.

FAC11858;LOP1185801,LOP1185802; SRF11870 + .

FAC11851;LOP1185101; SRF11871 + .

FAC11872;LOP1187201,LOP1187202,LOP1187203,LOP1187204; SRF11873 + .

FAC11874;LOP1187401; SRF11875 +.

FAC11876;LOP1187601,LOP1187602; SRF11877 + .

FAC11878;LOP1187801; SRF11879 + .

FAC11880;LOP1188001; SRF11881 + .

/END_FACES

/LOOPS

LOP1211901;EDG12937+,EDG12895+,EDG12935-,EDG13199-.

LOP1212101;EDG13167+,EDG12959+,EDG12897+,EDG13201-.

LOP1212301;EDG13203+,EDG13205+,EDG12907+,EDG13131-.

LOP1212501;EDG12909+,EDG13207-,EDG13209-,EDG13001+.

LOP1212701;EDG13169+,EDG13201+,EDG12917+,EDG12991+.

LOP1212901;EDG13213-,EDG13211-.

LOP1212902;EDG12921-,EDG12925-.

LOP1213101;EDG13217-,EDG13215-.

LOP1213102;EDG12929-,EDG12933-.

LOP1213301;EDG13219+,EDG12939+,EDG13221-,EDG13223-.

LOP1213501;EDG13227-,EDG12955-,EDG12967-,EDG13225-,EDG12945-,

EDG12943-.

LOP1213502;EDG13231-,EDG13229-.

LOP1213701;EDG12951+,EDG13233-,EDG13235+,EDG13237+. 
LOP1213901;EDG12953+,EDG13237-,EDG13239+,EDG13233+.

LOP1214101;EDG12983+,EDG13241+,EDG12965-,EDG13149-.

LOP1214301;EDG12969+,EDG13243-,EDG13245+,EDG13247+.

/END_LOOPS

IEDGES

EDG12441;VTX13739,VTX13727; CRV12442 +.

EDG12443;VTX13727,VTX13734; CRV12444 +.

EDG12445;VTX13734,VTX13740; CRV12446 +.

EDG12447;VTX13740,VTX13736; CRV12448 +.

EDG12449;VTX13736,VTX13716; CRV12450 +.

EDG12451;VTX13738,VTX13737; CRV12452 +.

EDG12453;VTX13737,VTX13738; CRV12454 +.

EDG12455;VTX13725,VTX13736; CRV12456 +.

EDG12457;VTX13725,VTX13726; CRV12458 +.

EDG12459;VTX13724,VTX13725; CRV12460 +.

EDG12461;VTX13723,VTX13724; CRV12462 +.

EDG12463;VTX13730,VTX13732; CRV12464 +.

EDG12465;VTX13730,VTX13720; CRV12466 +.

EDG12467;VTX13720,VTX13719; CRV12468 +.

EDG12469;VTX13719,VTX13733; CRV12470 +.

/END_EDGES

NVERTICES

VTX14074;PNT140740.

VTX14077;PNT140770.

VTX14091;PNT140910.

VTX14090;PNT140900.

VTX14095;PNT140950.

VTX14094;PNT140940.

VTX14112;PNT141120.

VTX14115;PNT141150.

VTX13755;PNT137550.

VTX13748;PNT137480.

VTX13783;PNT137830.

VTX13770;PNT137700.

VTX13778:PNT137780.

VTX13767;PNT137670.

VTX13784;PNT137840.

VTX14075;PNT140750. 
/END_VERTICES

IEND_TOPOLOGY

/GEOMETRY

ISURFACES

SRF11907; CYLINDER; PNT119071; UNV117340; 0.12850013.

SRF11908; CYLINDER; PNT119071; UNV117340; 0.12850013.

SRF11910; PLANE; UNV117340; 1.18700004.

SRF11912; CONE; PNT119121; UNV117440; -0.94405442.

SRF11914; CYLINDER; PNT118661; UNV117340; 0.93750000.

SRF11916; CYLINDER; PNT118661; UNV117340; 0.87500000.

SRF12348; PLANE; UNV117440; -0.12200000 .

SRF12350; CYLINDER; PNT134680; UNV117440; 0.65649998.

SRF12352; CYLINDER; PNT134680; UNV117440; 0.65649998.

SRF12354; PLANE; UNV117440; -0.06200000 .

SRF12356; PLANE; UNV117340; 0.59299999.

SRF12358; PLANE; UNV117340; 0.59299999.

SRF12360; CONE; PNT138920; UNV117340; 0.51795375.

SRF12362; CONE; PNT138920; UNV117340; 0.51795375.

SRF12364; CONE; PNT138930; UNV117340; 0.51795363.

SRF12366; CONE; PNT138930; UNV117340; 0.51795363.

SRF12368; CYLINDER; PNT124080; UNV117340; 1.83700001.

SRF12370; CYLINDER; PNT124080; UNV117340; 2.77699995.

SRF12372; PLANE; UNV117440; -0.12200000 .

SRF12374; PLANE; UNV117340; 0.43700001.

SRF12376; CYLINDER; PNT124080; UNV117340; 1.83700001.

IEND_SURFACES

ICURVES

CRV11734; CIRCLE; PNT117340; UNV117340; PNT117341.

CRV11736; BSPLINE; 3; 24;PNT11736001,PNT11736002,PNT11736003,

PNT11736004,PNT11736005,PNT11736006,PNT11736007,PNT11736008,

PNT11736009,PNT11736010,PNT11736011,PNT11736012,PNT11736013,

PNT11736014,PNT11736015,PNT11736016,PNT11736017,PNT11736018,

PNT11736019,PNT11736020,PNT11736021,PNT11736022,PNT11736023,

PNT11736024.

CRV13296: LINE; PNT139360; UNV117340.

CRV13302; CIRCLE; PNT133020; UNV117340; PNT139230.

CRV13304; LINE; PNT139230; UNV117340.

CRV13306; LINE; PNT139240; UNV117340. 
CRV13308; CIRCLE; PNT133020; UNV117340; PNT133081.

CRV13310; BSPLINE; 3; 42;PNT13310001,PNT13310002,PNT13310003,

PNT13310004,PNT13310005,PNT13310006,PNT13310007,PNT13310008,

PNT13310009,PNT13310010,PNT13310011,PNT13310012,PNT13310013, PNT13310014,PNT13310015,PNT13310016,PNT13310017,PNT13310018, PNT13310019,PNT13310020,PNT13310021,PNT13310022,PNT13310023, PNT13310024,PNT13310025,PNT13310026,PNT13310027,PNT13310028, PNT13310029,PNT13310030,PNT13310031,PNT13310032,PNT13310033, PNT13310034,PNT13310035,PNT13310036,PNT13310037,PNT13310038, PNT13310039,PNT13310040,PNT13310041,PNT13310042.

CRV13312; CIRCLE; PNT133020; UNV117340; PNT139240.

CRV13314; BSPLINE; 3; 16;PNT13314001,PNT13314002,PNT13314003,

PNT13314004,PNT13314005,PNT13314006,PNT13314007,PNT13314008,

PNT13314009,PNT13314010,PNT13314011,PNT13314012,PNT13314013, PNT13314014,PNT13314015,PNT13314016.

CRV13316; CIRCLE; PNT133160; UNV117340; PNT139200.

CRV13324; CIRCLE; PNT133160; UNV117340; PNT133241.

CRV13412; CIRCLE; PNT134020; UNV117340; PNT134121.

CRV13414; BSPLINE; 3; 16;PNT13414001,PNT13414002,PNT13414003,

PNT13414004,PNT13414005,PNT13414006,PNT13414007,PNT13414008,

PNT13414009,PNT13414010,PNT13414011,PNT13414012,PNT13414013,

PNT13414014,PNT13414015,PNT13414016.

CRV13416; CIRCLE; PNT134160; UNV117340; PNT139330.

CRV13418; LINE; PNT139330; UNV117340.

CRV13420; LINE; PNT139990; UNV117340.

CRV13422; BSPLINE; 3; 29;PNT13422001,PNT13422002,PNT13422003,

PNT13422004,PNT13422005,PNT13422006,PNT13422007,PNT13422008,

PNT13422009,PNT13422010,PNT13422011,PNT13422012,PNT13422013,

PNT13422014,PNT13422015,PNT13422016,PNT13422017,PNT13422018,

PNT13422019,PNT13422020,PNT13422021,PNT13422022,PNT13422023,

PNT13422024,PNT13422025,PNT13422026,PNT13422027,PNT13422028,

PNT13422029.

CRV13424; CIRCLE; PNT134160; UNV117340; PNT134241,

IEND_CURVES

IPOINTS

PNT12694007; 1.03147268, -1.88477874, 2.65294743.

PNT12694008; $1.02363145,-1.88648355,2.64048505$

PNT12694009; $1.01508069,-1.88823354,2.62891936$.

PNT12694010; $1.00586081,-1.88999414,2.61816525$. 
PNT12694011; 0.99601489, - - .89173067, 2.60823250

PNT12694020; 0.88674426, -1.90130079, 2.55522299.

PNT 12694021; $0.87319481,-1.90126789,2.55334210$

PNT 12694022; $0.85955244,-1.90095474,2.55223274$.

PNT12694023; 0.85042524, -1.90058005, 2.55199599.

PNT12694024; $0.84586364,-1.90034223,2.55200005$.

PNT12720001; 0.84609222, -1.90035415, 2.95799994.

PNT12720002; 0.85065025, -1.90059030, 2.95799994 .

PNT12720003; 0.85976994, -1.90097165, 2.95775509.

PNT 12720004; 0.87340039, -1.90127027, 2.95663476 .

PNT12720014; 0.99608731, -1.89171839, 2.90169883. ...

IEND_POINTS

UNIT_VECTORS

UNV120460; $0.99452192,0.10452847,0.00000000$.

UNV $120500 ; 0.74314481,-0.66913062,0.00000000$.

UNV120540; $-0.30901700,-0.95105654,0.00000000$.

UNV120940; $-0.20791164,0.97814763,0.00000000$.

UNV120980;0.80901700, 0.58778524, 0.00000000 .

UNV121220;0.20791170, 0.97814763, 0.00000000 .

UNV121260; $-0.80901700,0.58778524,0.00000000$.

IEND_UNIT_VECTORS

/END_GEOMETRY

/END_PART_MODEL 


\section{REFERENCES}

[Barkmeyer86]

[Fowler88]

[Hopp87a]

[Hopp87b]

[Lee88]

[Ressler87]

[Smith88]
Barkmeyer, E., Mitchell, M., Mikkilineni, K., Su, S., Lam, H., "An Architecture for Distributed Data Management in Computer Integrated Manufacturing," NBSIR 86-3312, National Bureau of Standards, Gaithersburg, MD, January 1986.

Fowler, J.E., "MDP/Part Model Working Group Activity Meeting Summary," Memo, 21 April 1988.

Hopp, T.H., Tu, J.S., "Part Geometry Data in the AMRF," NBSIR 87-3551, National Bureau of Standards, Gaithersburg, MD, April 1987.

Hopp, T.H., "AMRF Database Report Format: Part Model," NBSIR 87-3672, National Bureau of Standards, Gaithersburg, MD, September 1987.

Lee, Y.T., Ressler, S., "Converting the AMRF Part Model Report to a PDES/STEP Subset," NBSIR 88-3818, National Bureau of Standards, Gaithersburg, MD, July 1988.

Ressler, S., "Using The AMRF Part Model Report," NBSIR 873531, National Bureau of Standards, Gaithersburg, MD, February 1987.

Smith, B., Rinaudot, G.. eds., "Product Data Exchange Specification," NISTIR 88-4004, National Institute of Standards and Technology, Gaithersburg, MD, December 1988. 
NIST-114A

(REV. 3-89)
U.S. DEPARTMENT OF COMMERCE

NATIONAL INSTITUTE OF STANDARDS AND TECHNOLOGY

\section{BIBLIOGRAPHIC DATA SHEET}

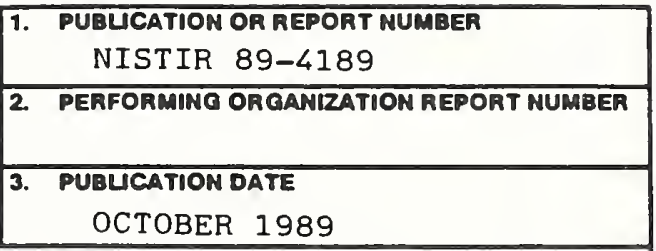

4. TITLE AND SUBTITLE

AMRF Part Model Extensions

5. AUTHOR(S)

Allison Barnard

6. PERFORMINO ORGANIZATION (IF JOINT OR OTHER THAN NIST, SEE INSTRUCTIONS)

U.S. DEPARTMENT OF COMMERCE

MATIONAL INSTITUTE OF STANDARDS AND TECHNOLOGY

OATTHERSBURG, MD 20899

7. CONTAACT/GAANT MUMBER

8. TYPE OF REPORT AND PEAIOD COVERED

9. SPONSOAINO ORQAMIZATION NAME AND COMPLETE ADDRESS (STREET, CITY, STATE, ZIP)

10. SUPPLEMENTARY MOTES

DOCUMENT DESCRIBES A COMPUTER PROGRAM; SF-185, FIPS SOFTWARE SUMMARY, IS ATTACHED.

11. ABSTRACT (A 200-WORD OR LESS FACTUAL SUMMARY OF MOST SIONIFICANT INFORMATION. IF DOCUMENT IMCLUDES A SIGNIFICANT BIBUOGAAPHY OR ITTERATURE SURVEY, MENTION IT HERE.)

12. KEY WORDS (6 TO 12 ENTRIES; ALPHABETICAL ORDER; CAPITALZE ONLY PROPER MAMES; AND SEPARATE KEY WORDS BY SEMICOLONS)

This document specifies the addition of ellipses, hyperbolas, parabolas, and b-splines to the AMRF Part Model Report Fonmat. These reports are used throughout the AMRF to communicate part model data between application processes and the global AMRF database. Part model data consists of geometry, topology, features, and tolerances. This document is intended to be used by programmers implementing systems that make use of AMRF part model data. While this report is a complete description of the extensions, the document "AMRF Database Report Format: Part Model" by T.H. Hopp (NBSIR 87-3672) is also required to complete the gramar.

AMRF, boundary representation, format languages, geometry, PDES, solid models, topology.

FOR OFFICIAL DISTAIBUTION. DO NOT AELEASE TO NATIONAL TECHNICAL INFOAMATION SERVICE (MTIS).

ORDER FROM SUPERINTENDENT OF DOCUMENTS, U.S. COVERMMENT PRINTING OFFICE, WASHINGTON, DC 20402.

ORDER FROM NATIONGL TECHMICAL INFORMATION SERVGE (NTIS), SPAMOFIELD, VA 22161.

14. NUMBER OF PRINTED PAGES 
